Acta Crystallographica Section D

Biological

Crystallography

ISSN 0907-4449

\section{Hongliang Xu}

Mathematics Department, SUNY College at Buffalo, 1300 Elmwood Avenue, Buffalo, NY 14222, USA, and Hauptman-Woodward Medical Research Institute, 700 Ellicott Street Buffalo, NY 14203, USA

Correspondence e-mail: xu@hwi.buffalo.edu

\section{Enhancing MAD $F_{\mathrm{A}}$ data for substructure determination}

Heavy-atom substructure determination is a critical step in phasing an unknown macromolecular structure. Dual-space (Shake-and-Bake) recycling is a very effective procedure for locating the substructure (heavy) atoms using $F_{\mathrm{A}}$ data estimated from multiple-wavelength anomalous diffraction. However, the estimated $F_{\mathrm{A}}$ are susceptible to the accumulation of errors in the individual intensity measurements at several wavelengths and from inaccurate estimation of the anomalous atomic scattering corrections $f^{\prime}$ and $f^{\prime \prime}$. In this paper, a new statistical and computational procedure which merges multiple $F_{\mathrm{A}}$ estimates into an averaged data set is used to further improve the quality of the estimated anomalous amplitudes. The results of $18 \mathrm{Se}$-atom substructure determinations provide convincing evidence in favor of using such a procedure to locate anomalous scatterers.

\section{Introduction}

MAD (multiple-wavelength anomalous diffraction) is one of the primary techniques used to solve macromolecular structures. In a MAD experiment, two or more wavelengths are used to measure anomalous dispersion. The determination of a new protein structure is typically a two-step process. The first step is to locate the anomalous scatterers; the positions of the substructure atoms are then used as a bootstrap to initiate the phasing of the complete structure. Substructure atoms can be located using computational procedures that are based on either Patterson or direct methods. In either case, substructure intensities are estimated from MAD data by using either the so-called $F_{\mathrm{A}}$ formula, anomalous differences or dispersive differences, or various combinations thereof.

\subsection{The analysis of MAD data}

Let $F_{\mathrm{P}}, F_{\mathrm{D}}$ and $F_{\mathrm{A}}$ be the structure-factor amplitudes of the native protein structure, the derivative structure and the substructure, and $\varphi_{\mathrm{P}}$ and $\varphi_{\mathrm{A}}$ be the native and substructure phases, respectively. Karle (1980) and Hendrickson et al. (1985) showed by algebraic analysis that for a given wavelength $\lambda$,

$$
\begin{aligned}
{ }^{\lambda} F_{\mathrm{D}}^{ \pm 2} & =F_{\mathrm{P}}^{2}+a_{\lambda} F_{\mathrm{A}}^{2}+b_{\lambda} F_{\mathrm{P}} F_{\mathrm{A}} \cos (\Delta \varphi) \pm c_{\lambda} F_{\mathrm{P}} F_{\mathrm{A}} \sin (\Delta \varphi), \quad(1) \\
a_{\lambda} & =\left[\left(^{\lambda} f^{\prime}\right)^{2}+\left({ }^{\lambda} f^{\prime \prime}\right)^{2}\right] / f_{0}^{2}, \quad b_{\lambda}=2\left(^{\lambda} f^{\prime}\right) / f_{0}, \quad c_{\lambda}=2\left({ }^{\lambda} f^{\prime \prime}\right) / f_{0}, \\
\Delta \varphi & =\varphi_{\mathrm{P}}-\varphi_{\mathrm{A}}
\end{aligned}
$$

where $f_{0}$ is the normal atomic scattering factor, ${ }^{\lambda} f^{\prime}$ and ${ }^{\lambda} f^{\prime \prime}$ are the anomalous scattering corrections (wavelength-dependent) and the '+' sign refers to reflection $(h, k, l)$ and the '-' sign to reflection $(-h,-k,-l)$.
Received 1 April 2010 Accepted 30 June 2010
(C) 2010 International Union of Crystallography Printed in Singapore - all rights reserved 
Table 1

Selenium-substructure data sets used in this investigation.

\begin{tabular}{|c|c|c|c|c|c|}
\hline \multirow[b]{2}{*}{$\begin{array}{l}\text { PDB } \\
\text { code }\end{array}$} & \multicolumn{2}{|l|}{ Selenium sites } & \multirow[b]{2}{*}{$\begin{array}{l}\text { Space } \\
\text { group }\end{array}$} & \multirow{2}{*}{$\begin{array}{l}\text { MAD } \\
\text { resolution } \\
(\AA)\end{array}$} & \multirow[b]{2}{*}{ Reference } \\
\hline & Theoretical $\dagger$ & Actual & & & \\
\hline $1 q c z$ & 5 & 4 & $I 422$ & 2.0 & Mathews et al. (1999) \\
\hline $1 \mathrm{bx} 4$ & 8 & 7 & $P 2_{1} 2_{1} 2$ & 2.3 & Mathews et al. (1998) \\
\hline $1 \mathrm{cb} 0$ & 9 & 8 & P321 & 2.2 & Appleby et al. (1999) \\
\hline $1 \mathrm{t} 5 \mathrm{~h}$ & 10 & 10 & $P 3_{2} 21$ & 2.5 & Gulick et al. (2004) \\
\hline 1gso & 13 & 13 & $P 2_{1} 2_{1} 2_{1}$ & 2.2 & Wang et al. (1998) \\
\hline $1 \mathrm{jxh}$ & 14 & 14 & $P 4_{1} 2{ }_{1} 2$ & 3.1 & Cheng et al. (2002) \\
\hline $1 \mathrm{dbt}$ & 21 & 19 & $P 2{ }_{1} 2{ }_{1}$ & 2.5 & Appleby et al. (2000) \\
\hline 1jen & 24 & 22 & $P 2_{1}$ & 2.3 & Ekstrom et al. (1999) \\
\hline $1 \mathrm{jc} 4$ & 28 & 24 & $P 2_{1}$ & 2.1 & McCarthy et al. (2001) \\
\hline $1 \mathrm{cli}$ & 28 & 28 & $P 2_{1} 2_{1} 2_{1}$ & 3.0 & Li et al. (1999) \\
\hline $1 \mathrm{a} 7 \mathrm{a}$ & 32 & 30 & $C 222$ & 2.8 & Turner et al. (1998) \\
\hline $118 \mathrm{a}$ & 42 & 40 & $P 2_{1}$ & 2.6 & Arjunan et al. (2002) \\
\hline $1 \mathrm{e} 3 \mathrm{~m}$ & 48 & 45 & $P 2_{1} 2_{1} 2_{1}$ & 3.0 & Lamers et al. (2000) \\
\hline 1hi8 & 50 & 50 & $P 3_{2}$ & 3.0 & Butcher et al. (2001) \\
\hline $1 \mathrm{~m} 32$ & 66 & 66 & $P 2_{1}$ & 2.6 & Chen et al. (2002) \\
\hline $1 \mathrm{dq} 8$ & 68 & 60 & $P 2_{1}$ & 2.6 & Istvan et al. (2000) \\
\hline $1 e 2 y$ & 70 & 60 & $P 2_{1}$ & 3.2 & Alphey et al. (2000) \\
\hline 1eq2 & 70 & 70 & $P 2_{1}$ & 3.0 & Deacon et al. (2000) \\
\hline
\end{tabular}

$\dagger$ Potential sites based on the amino-acid sequence. $\ddagger$ Number of sites reported in the published protein structure.

Each of the measurements of $F_{\mathrm{D}}^{ \pm}$at a given wavelength gives us two equations (1) and the different wavelengths may be treated as a system of simultaneous equations. For two or more wavelengths, (1) represents an overdetermined system of equations that can be analyzed to obtain values of $F_{\mathrm{A}}, F_{\mathrm{P}}$ and $\Delta \varphi$ for each reflection. Once $F_{\mathrm{A}}$ estimates are available, direct methods can be applied to locate anomalous scatterers. Subsequently, substructure phases, $\varphi_{\mathrm{A}}$, can be calculated from the refined substructure. The initial experimental map is then calculated from estimated $F_{\mathrm{P}}$ and protein structure phases $\varphi_{\mathrm{P}}=\Delta \varphi+\varphi_{\mathrm{A}}$.

\section{2. $F_{\mathrm{A}}$ data estimation}

In a typical MAD experiment, three wavelengths $\left(\lambda_{1}\right.$, edge; $\lambda_{2}$, peak; $\lambda_{3}$, remote) are used to measure Bijvoet differences. The common procedure in protein crystallography is to use ALL measured data to estimate the substructure structure factors $F_{\mathrm{A}}$. In theory, these $F_{\mathrm{A}}$ values should be closer to the true substructure factors than those estimated from singlewavelength or two-wavelength anomalous dispersion data would be.

From a mathematical point of view, at least two wavelengths [four equations (1)] are needed to estimate the three unknown quantities $F_{\mathrm{A}}, F_{\mathrm{P}}$ and $\Delta \varphi$. When three-wavelength data are available, we could have four different $F_{\mathrm{A}}$ estimates from four different inputs: $\lambda_{1}$ and $\lambda_{2}, \lambda_{1}$ and $\lambda_{3}, \lambda_{2}$ and $\lambda_{3}$, and $\lambda_{1}, \lambda_{2}$ and $\lambda_{3}$ (common protocol). In theory, all the $F_{\mathrm{A}}$ estimates should be the same if the anomalous dispersion data are error-free. Unfortunately, the $F_{\mathrm{A}}$ values can be estimated only approximately and their accuracy depends on many factors, including the precision of intensity measurements at various wavelengths, the stability of the wavelengths during the whole data-collection session, the effect of crystal deterioration etc.
Estimated $F_{\mathrm{A}}$ values can be divided into three classes: reliably estimated, overestimated or underestimated. If the number of significantly overestimated $F_{\mathrm{A}}$ is large, direct methods such as Shake-and-Bake will fail. From a statistical point of view, data averaged over four $F_{\mathrm{A}}$ estimates will reduce the number of overestimated reflections and should be better than any individual $F_{\mathrm{A}}$ estimates. To test our hypothesis, we carried out a series of computational experiments described in the next section.

\section{Materials and methods}

The relative merits of using different $F_{\mathrm{A}}$ estimates have been determined by a postmortem analysis of the Shake-and-Bake procedure (Miller et al., 1994; Weeks \& Miller, 1999) for 18 known SeMet protein substructures ranging in size from five to 70 Se sites in the asymmetric unit. Basic information such as the Protein Data Bank (PDB) code, number of Se atoms in the asymmetric unit $\left(N_{\mu}\right)$, space group and MAD data resolution is listed in Table 1 for these substructures. In each case, three wavelengths of anomalous dispersion data were available and the SHELXC program (Sheldrick, 2008) was used to calculate four sets of substructure $F_{\mathrm{A}}$ values using the four different kinds of input mentioned in the previous section. Each set of $F_{\mathrm{A}}$ data was then normalized using a modified version of the SHELXD program (Schneider \& Sheldrick, 2002) to output the normalized substructure structure factors $E_{\mathrm{A}}$.

All sets of normalized substructure structure factors were truncated to $3 \AA$ resolution, the remaining reflections were sorted in decreasing order according to their $E_{\mathrm{A}}$ values and the top $30 N_{\mu}$ reflections were then selected to generate the $300 N_{\mu}$ most reliable three-phase structure invariants for $\operatorname{SnB}$ (Weeks $\&$ Miller, 1999) applications. Samples of 1000 randomly positioned $N_{\mu}$-atom trial structures were generated for each set of test data and subjected to $2 N_{\mu}$ cycles of $\operatorname{Sn} B$ dual-space refinement. Following refinement, the mean phase error (MPE) relative to the known substructure was determined for each trial structure and trials with MPE values of less than $30^{\circ}$ were counted as solutions. In all cases, low MPE values were perfectly correlated with low values of the minimal function. The success rate, defined as the percentage of trial structures that converged to solution at the end of a fixed number of $\operatorname{SnB}$ cycles, provided an important indication of the quality of the chosen computational method. The latest version of the $\operatorname{SnB}$ program implementing the statistical minimal function $(\mathrm{Xu} \&$ Hauptman, 2004; Xu et al., 2005) was used to obtain the computational results reported in this paper.

\section{Results}

\subsection{Results from individual $\boldsymbol{F}_{\mathrm{A}}$ estimations}

The success rates obtained from statistical Shake-and-Bake using each of the four $E_{\mathrm{A}}$ data sets for the 18 Se-atom test substructures are listed in Table 2 under the headings $E_{\mathrm{A}}\left(\lambda_{1}, \lambda_{3}\right), E_{\mathrm{A}}\left(\lambda_{2}, \lambda_{3}\right), E_{\mathrm{A}}\left(\lambda_{1}, \lambda_{2}\right)$ and $E_{\mathrm{A}}\left(\lambda_{1}, \lambda_{2}, \lambda_{3}\right)$, respectively, for the four different ways of generating $E_{\mathrm{A}}$ values. 
Table 2

Comparative $S n B$ success rates for the 18 Se-atom substructures with four different $E_{\mathrm{A}}$ data sets and their averaged data set $\left\langle E_{\mathrm{A}}\right\rangle$.

Statistically higher success rates when compared with those of the $E_{\mathrm{A}}\left(\lambda_{1}, \lambda_{2}\right.$, $\lambda_{3}$ ) data sets are highlighted in bold.

\begin{tabular}{lcccccr}
\hline & \multicolumn{5}{l}{ Success rate $(\%)$} \\
\cline { 3 - 7 } PDB & Se & & & & \\
code & sites & $E_{\mathrm{A}}\left(\lambda_{1}, \lambda_{3}\right)$ & $E_{\mathrm{A}}\left(\lambda_{2}, \lambda_{3}\right)$ & $E_{\mathrm{A}}\left(\lambda_{1}, \lambda_{2}\right)$ & $E_{\mathrm{A}}\left(\lambda_{1}, \lambda_{2}, \lambda_{3}\right)$ & $\left\langle E_{\mathrm{A}}\right\rangle$ \\
\hline 1qcz & 4 & 14.2 & 13.7 & 15.6 & 14.7 & 16.8 \\
1bx4 & 7 & 11.7 & 12.4 & 9.3 & 12.4 & 11.7 \\
1cb0 & 8 & $\mathbf{6 . 9}$ & 1.8 & $\mathbf{5 . 7}$ & 2.9 & 3.5 \\
1t5h & 10 & 2.8 & 4.0 & 1.3 & 3.7 & 3.0 \\
1 gso & 13 & 7.9 & 5.1 & 5.1 & 6.6 & 6.2 \\
1jxh & 14 & 0.0 & 0.0 & 0.0 & 0.0 & $\mathbf{0 . 8}$ \\
1dbt & 19 & 5.4 & 2.5 & $\mathbf{9 . 0}$ & 5.8 & $\mathbf{8 . 1}$ \\
1jen & 22 & 14.3 & 12.6 & 11.9 & 11.9 & 13.2 \\
1jc4 & 24 & 24.9 & 23.8 & 12.7 & 32.7 & 32.2 \\
1cli & 28 & $\mathbf{2 . 1}$ & 1.2 & 0.0 & 0.7 & $\mathbf{4 . 9}$ \\
1a7a & 30 & 4.1 & 4.4 & 2.6 & 5.2 & 5.6 \\
118a & 40 & 5.7 & 2.0 & 0.0 & 12.9 & $\mathbf{2 5 . 6}$ \\
1e3m & 45 & 4.6 & 4.3 & 4.3 & 5.7 & 7.6 \\
1hi8 & 50 & 0.0 & 1.3 & $\mathbf{4 1 . 3}$ & 2.9 & $\mathbf{1 9 . 6}$ \\
1m32 & 66 & 1.3 & 1.4 & 0.0 & 2.6 & $\mathbf{6 . 6}$ \\
1dq8 & 60 & $\mathbf{1 6 . 9}$ & 2.2 & $\mathbf{1 9 . 3}$ & 12.1 & $\mathbf{1 6 . 3}$ \\
1e2y & 70 & 0.5 & 0.5 & 2.3 & 3.9 & 2.8 \\
1eq2 & 70 & 0.1 & 0.0 & 0.0 & 0.1 & $\mathbf{0 . 8}$ \\
\hline Average & 6.9 & 5.2 & 7.4 & 7.6 & 10.3 \\
\hline
\end{tabular}

We have observed from the first four columns of Table 2 that (i) five $E_{\mathrm{A}}\left(\lambda_{1}, \lambda_{3}\right)$ data sets, two $E_{\mathrm{A}}\left(\lambda_{2}, \lambda_{3}\right)$ data sets, four $E_{\mathrm{A}}\left(\lambda_{1}, \lambda_{2}\right)$ data sets and eight $E_{\mathrm{A}}\left(\lambda_{1}, \lambda_{2}, \lambda_{3}\right)$ data sets yielded the highest success rates, (ii) three $E_{\mathrm{A}}\left(\lambda_{1}, \lambda_{3}\right)$ data sets, nine $E_{\mathrm{A}}\left(\lambda_{2}, \lambda_{3}\right)$ data sets, $12 E_{\mathrm{A}}\left(\lambda_{1}, \lambda_{2}\right)$ data sets and two $E_{\mathrm{A}}\left(\lambda_{1}, \lambda_{2}\right.$, $\lambda_{3}$ ) data sets yielded the lowest success rates and (iii) none of the four $E_{\mathrm{A}}$ estimates produced solutions for all 18 substructures. In fact, two $E_{\mathrm{A}}\left(\lambda_{1}, \lambda_{3}\right)$ data sets (1jxh and 1hi8), two $E_{\mathrm{A}}\left(\lambda_{2}, \lambda_{3}\right)$ data sets (1jxh and 1eq2), five $E_{\mathrm{A}}\left(\lambda_{1}, \lambda_{2}\right)$ data sets (1jxh, 1cli, 118a, 1m32 and 1eq2) and one $E_{\mathrm{A}}\left(\lambda_{1}, \lambda_{2}, \lambda_{3}\right)$ data set (1jxh) failed to yield solutions. Overall, $E_{\mathrm{A}}\left(\lambda_{1}, \lambda_{2}, \lambda_{3}\right)$ data sets produced most of the highest success rates and the smallest number of failures. The results confirm why $E_{\mathrm{A}}\left(\lambda_{1}, \lambda_{2}\right.$, $\left.\lambda_{3}\right)$ is chosen as a common protocol in protein crystallography to estimate the substructure structure factors.

\subsection{Effects of the averaged $\boldsymbol{F}_{\mathrm{A}}$ estimations}

Since it is impossible to eliminate experimental errors completely or to predict which $E_{\mathrm{A}}$ estimates will fail to produce solutions, we can utilize statistical procedures to identify reflections with the most reliably estimated substructure structure factors. From a statistical point of view, each of the four data sets can be regarded as independent estimates of the substructure structure factors and the values of the averaged $\left\langle E_{\mathrm{A}}\right\rangle$ data set should be more reliable than any of the individual estimates. To verify this hypothesis, all reflections in the averaged data set were sorted in decreasing order of the $\left\langle E_{\mathrm{A}}\right\rangle$ values and the top $30 N_{\mu}$ reflections were selected as input to statistical Shake-and-Bake. The success rates for the $18 \mathrm{Se}$-atom test substructures are listed in Table 2 under the heading $\left\langle E_{\mathrm{A}}\right\rangle$. When comparing two success rates $(x$ and $y$ ) obtained from two different data sets, $y$ is statistically
Table 3

Comparative $S n B$ success rates for the 18 Se-atom substructures with different $E_{\mathrm{A}}$ data sets and different reflection sortings..

Statistically higher success rates yielded from $E_{\mathrm{A}}$ and $\left\langle E_{\mathrm{A}}\right\rangle$ reflection sortings are highlighted in bold.

\begin{tabular}{|c|c|c|c|c|c|}
\hline \multirow[b]{2}{*}{$\begin{array}{l}\text { PDB } \\
\text { code }\end{array}$} & \multirow[b]{2}{*}{$\begin{array}{l}\text { Reflection } \\
\text { sorting }\end{array}$} & \multicolumn{4}{|c|}{ Success rate $(\%)$} \\
\hline & & $E_{\mathrm{A}}\left(\lambda_{1}, \lambda_{3}\right)$ & $E_{\mathrm{A}}\left(\lambda_{2}, \lambda_{3}\right)$ & $E_{\mathrm{A}}\left(\lambda_{1}, \lambda_{2}\right)$ & $E_{\mathrm{A}}\left(\lambda_{1}, \lambda_{2}, \lambda_{3}\right)$ \\
\hline \multirow[t]{2}{*}{$1 \mathrm{qcz}$} & $E_{\mathrm{A}}$ & 14.2 & 13.7 & 15.6 & 14.7 \\
\hline & $\left\langle E_{\mathrm{A}}\right\rangle$ & 16.1 & 16.9 & 18.5 & 16.0 \\
\hline \multirow[t]{2}{*}{$1 \mathrm{bx} 4$} & $E_{\mathrm{A}}$ & 11.7 & 12.4 & 9.3 & 12.4 \\
\hline & $\left\langle E_{\mathrm{A}}\right\rangle$ & 12.9 & 11.9 & 11.4 & 12.2 \\
\hline \multirow[t]{2}{*}{$1 \mathrm{cb} 0$} & $E_{\mathrm{A}}$ & 6.9 & 1.8 & 5.7 & 2.9 \\
\hline & $\left\langle E_{\mathrm{A}}\right\rangle$ & 4.4 & 2.5 & 3.4 & 4.0 \\
\hline \multirow[t]{2}{*}{$1 \mathrm{t} 5 \mathrm{~h}$} & $E_{\mathrm{A}}$ & 2.8 & 4.0 & 1.3 & 3.7 \\
\hline & $\left\langle E_{\mathrm{A}}\right\rangle$ & 3.4 & 3.1 & 3.7 & 2.5 \\
\hline \multirow[t]{2}{*}{ 1gso } & $E_{\mathrm{A}}$ & 7.9 & 5.1 & 5.1 & 6.6 \\
\hline & $\left\langle E_{\mathrm{A}}\right\rangle$ & 6.7 & 5.9 & 6.5 & 6.6 \\
\hline \multirow[t]{2}{*}{$1 \mathrm{jxh}$} & $E_{\mathrm{A}}$ & 0.0 & 0.0 & 0.0 & 0.0 \\
\hline & $\left\langle E_{\mathrm{A}}\right\rangle$ & 0.6 & 1.1 & 1.3 & 0.9 \\
\hline \multirow[t]{2}{*}{$1 \mathrm{dbt}$} & $E_{\mathrm{A}}$ & 5.4 & 2.5 & 9.0 & 5.8 \\
\hline & $\left\langle E_{\mathrm{A}}\right\rangle$ & 8.8 & 7.2 & 9.0 & 8.5 \\
\hline \multirow[t]{2}{*}{$1 \mathrm{jen}$} & $E_{\mathrm{A}}$ & 14.3 & 12.6 & 11.9 & 11.9 \\
\hline & $\left\langle E_{\mathrm{A}}\right\rangle$ & 14.0 & 13.4 & 13.7 & 14.7 \\
\hline \multirow[t]{2}{*}{$1 \mathrm{jc} 4$} & $E_{\mathrm{A}}$ & 24.9 & 23.8 & 12.7 & 32.7 \\
\hline & $\left\langle E_{\mathrm{A}}\right\rangle$ & 32.7 & 32.0 & 31.9 & 32.5 \\
\hline \multirow[t]{2}{*}{ 1cli } & $E_{\mathrm{A}}$ & 2.1 & 1.2 & 0.0 & 0.7 \\
\hline & $\left\langle E_{\mathrm{A}}\right\rangle$ & 5.0 & 5.0 & 4.7 & 5.3 \\
\hline \multirow[t]{2}{*}{$1 \mathrm{a} 7 \mathrm{a}$} & $E_{\mathrm{A}}$ & 4.1 & 4.4 & 2.6 & 5.2 \\
\hline & $\left\langle E_{\mathrm{A}}\right\rangle$ & 5.7 & 5.3 & 4.8 & 5.2 \\
\hline \multirow[t]{2}{*}{$118 \mathrm{a}$} & $E_{\mathrm{A}}$ & 5.7 & 2.0 & 0.0 & 12.9 \\
\hline & $\left\langle E_{\mathrm{A}}\right\rangle$ & 25.0 & 28.6 & 29.8 & 30.0 \\
\hline \multirow[t]{2}{*}{$1 \mathrm{e} 3 \mathrm{~m}$} & $E_{\mathrm{A}}$ & 4.6 & 4.3 & 4.3 & 5.7 \\
\hline & $\left\langle E_{\mathrm{A}}\right\rangle$ & 6.2 & 7.0 & 6.6 & 8.0 \\
\hline \multirow[t]{2}{*}{ 1hi8 } & $E_{\mathrm{A}}$ & 0.0 & 1.3 & 41.3 & 2.9 \\
\hline & $\left\langle E_{\mathrm{A}}\right\rangle$ & 10.8 & 17.6 & 23.4 & 18.1 \\
\hline \multirow[t]{2}{*}{$1 \mathrm{~m} 32$} & $E_{\mathrm{A}}$ & 1.3 & 1.4 & 0.0 & 2.6 \\
\hline & $\left\langle E_{\mathrm{A}}\right\rangle$ & 6.5 & 5.2 & 4.7 & 6.3 \\
\hline \multirow[t]{2}{*}{$1 \mathrm{dq} 8$} & $E_{\mathrm{A}}$ & 16.9 & 2.2 & 19.3 & 12.1 \\
\hline & $\left\langle E_{\mathrm{A}}\right\rangle$ & 18.9 & 18.6 & 17.1 & 17.1 \\
\hline \multirow[t]{2}{*}{$1 e 2 y$} & $E_{\mathrm{A}}$ & 0.5 & 0.5 & 2.3 & 3.9 \\
\hline & $\left\langle E_{\mathrm{A}}\right\rangle$ & 2.1 & 1.5 & 1.9 & 2.3 \\
\hline \multirow[t]{2}{*}{ 1eq2 } & $E_{\mathrm{A}}$ & 0.1 & 0.0 & 0.0 & 0.1 \\
\hline & $\left\langle E_{\mathrm{A}}\right\rangle$ & 1.0 & 1.1 & 1.2 & 1.1 \\
\hline
\end{tabular}

higher than $x$ if $y \geq x+2 \sigma(|y-x|)$, where $\sigma(x)$ is the standard deviation calculated by Bernoulli's distribution, $\sigma(x)=$ $[n x(1-x)]^{1 / 2}$, where $n$ is the number of trials, $x$ is the success rate expressed as a fraction and $\sigma(|y-x|)=\left[\sigma^{2}(x)+\sigma^{2}(y)\right]^{1 / 2}$, and $y$ is statistically lower than $x$ if $y \leq x-2 \sigma(|y-x|)$; otherwise $y$ is statistically equivalent to $x$. When compared with $E_{\mathrm{A}}\left(\lambda_{1}, \lambda_{2}, \lambda_{3}\right)$ data sets for the 18 test substructures, $\left\langle E_{\mathrm{A}}\right\rangle$ data sets yielded statistically higher success rates for eight test substructures (highlighted in bold in Table 2) and statistically equivalent success rates for the other ten test substructures. More importantly, $\left\langle E_{\mathrm{A}}\right\rangle$ data sets yielded solutions for all 18 test substructures.

\subsection{Effects of reflection selections}

As shown in Table 2, zero success rates were observed with at least one of the four estimated data sets for six test substructures (1jxh, 1cli, 118a, 1hi8, 1m32 and 1eq2), but not with their averaged data sets. There are two major differences between the individual $E_{\mathrm{A}}$ data sets and the $\left\langle E_{\mathrm{A}}\right\rangle$ data sets: (i) the amplitudes of the normalized substructure structure 
Table 4

Investigation of $E_{\mathrm{A}}$ estimates using different two-wavelength combinations for the selected substructures.

Possible causes for zero or near-zero $\operatorname{SnB}$ success rates are highlighted in bold.

\begin{tabular}{|c|c|c|c|c|c|c|}
\hline \multirow[b]{2}{*}{$\begin{array}{l}\text { PDB } \\
\text { code }\end{array}$} & \multirow{2}{*}{$\begin{array}{l}E_{\mathrm{A}} \\
\text { resolution } \dagger \\
(\AA)\end{array}$} & \multirow[b]{2}{*}{$\begin{array}{l}\text { No. of } \\
\text { reflections }\end{array}$} & \multirow[b]{2}{*}{$\begin{array}{l}\text { Reflection } \\
\text { sorting }\end{array}$} & \multirow{2}{*}{$\begin{array}{l}\text { Percentage } \\
\text { overestimates } \\
(\%)\end{array}$} & \multicolumn{2}{|c|}{ Correlation coefficient with $\left\langle E_{\mathrm{A}}\right\rangle$} \\
\hline & & & & & Overall & Overestimated \\
\hline \multirow[t]{3}{*}{$1 \mathrm{jxh}$} & $3.6(3.1)$ & 420 & $E_{\mathrm{A}}\left(\lambda_{1}, \lambda_{3}\right) \ddagger$ & 25 & 0.515 & 0.030 \\
\hline & & & $E_{\mathrm{A}}\left(\lambda_{2}, \lambda_{3}\right) \neq$ & 27 & 0.535 & -0.158 \\
\hline & & & $E_{\mathrm{A}}\left(\lambda_{1}, \lambda_{2}\right) \neq$ & 28 & 0.528 & -0.208 \\
\hline \multirow[t]{3}{*}{$1 \mathrm{cli}$} & $3.0(3.0)$ & 840 & $E_{\mathrm{A}}\left(\lambda_{1}, \lambda_{3}\right)$ & 41 & 0.387 & 0.100 \\
\hline & & & $E_{\mathrm{A}}\left(\lambda_{2}, \lambda_{3}\right)$ & 37 & 0.461 & 0.022 \\
\hline & & & $E_{\mathrm{A}}\left(\lambda_{1}, \lambda_{2}\right) \neq$ & 39 & 0.272 & -0.015 \\
\hline \multirow[t]{3}{*}{$118 \mathrm{a}$} & $2.8(2.6)$ & 1260 & $E_{\mathrm{A}}\left(\lambda_{1}, \lambda_{3}\right)$ & 47 & 0.242 & 0.002 \\
\hline & & & $E_{\mathrm{A}}\left(\lambda_{2}, \lambda_{3}\right)$ & 41 & 0.210 & 0.065 \\
\hline & & & $E_{\mathrm{A}}\left(\lambda_{1}, \lambda_{2}\right) \neq$ & 47 & 0.057 & -0.074 \\
\hline \multirow[t]{3}{*}{ 1hi8 } & $3.0(3.0)$ & 1500 & $E_{\mathrm{A}}\left(\lambda_{1}, \lambda_{3}\right) \neq$ & 50 & 0.234 & 0.050 \\
\hline & & & $E_{\mathrm{A}}\left(\lambda_{2}, \lambda_{3}\right)$ & 36 & 0.360 & 0.022 \\
\hline & & & $E_{\mathrm{A}}\left(\lambda_{1}, \lambda_{2}\right)$ & 38 & 0.331 & -0.045 \\
\hline \multirow[t]{3}{*}{$1 \mathrm{~m} 32$} & $3.0(2.6)$ & 1980 & $E_{\mathrm{A}}\left(\lambda_{1}, \lambda_{3}\right)$ & 35 & 0.285 & 0.000 \\
\hline & & & $E_{\mathrm{A}}\left(\lambda_{2}, \lambda_{3}\right)$ & 35 & 0.307 & 0.106 \\
\hline & & & $E_{\mathrm{A}}\left(\lambda_{1}, \lambda_{2}\right) \neq$ & 39 & 0.148 & 0.035 \\
\hline \multirow[t]{3}{*}{ 1eq2 } & $3.4(3.0)$ & 2100 & $E_{\mathrm{A}}\left(\lambda_{1}, \lambda_{3}\right) \neq$ & 44 & 0.260 & 0.012 \\
\hline & & & $E_{\mathrm{A}}\left(\lambda_{2}, \lambda_{3}\right) \neq$ & 39 & 0.313 & 0.048 \\
\hline & & & $E_{\mathrm{A}}\left(\lambda_{1}, \lambda_{2}\right) \neq$ & 40 & 0.259 & 0.086 \\
\hline
\end{tabular}

$\dagger$ Values in parentheses indicate the MAD data resolutions input to the SHELXC program. $\ddagger$ Data sets that yielded zero or near-zero $S n B$ success rates.

factors and (ii) the rankings of the reflections and thus the selection of the top reflections for $\operatorname{Sn} B$ phasing. To investigate the possible cause of the zero success rates, we replaced the amplitudes of the reflections in the $\left\langle E_{\mathrm{A}}\right\rangle$ data set with one of the four individually estimated $E_{\mathrm{A}}$ values, respectively, but kept the ranking of the reflections (no re-ranking). We then selected the top $30 N_{\mu}$ reflections as input to statistical Shakeand-Bake. The corresponding success rates, listed in Table 3, showed significant improvement in success rates. When compared with $E_{\mathrm{A}}$ ranking for the 18 test substructures, $\left\langle E_{\mathrm{A}}\right\rangle$ ranking yielded 35 statistically higher success rates (highlighted in bold in Table 3), 33 statistically equivalent success rates and only four statistically lower success rates. Furthermore, $\left\langle E_{\mathrm{A}}\right\rangle$ ranking yielded statistically higher success rates in all but one case for these six selected test substructures $(1 \mathrm{jxh}$, 1cli, 118a, 1hi8, 1m32 and 1eq2), thereby indicating that the selection of the top reflections for phasing is in fact the major factor in successful $\operatorname{Sn} B$ application. The large number of overestimated $E_{\mathrm{A}}$ amplitudes is the main reason for the low or near-zero success rates. The reflection ranking based on the $\left\langle E_{\mathrm{A}}\right\rangle$ amplitudes, rather than the $E_{\mathrm{A}}$ amplitudes, effectively eliminates the overestimated reflections and thus significantly improves the success of the Shake-and-Bake applications.

\subsection{Effects of measurement error}

It has been shown in the previous sections that the presence of overestimated $E \mathrm{~s}$ in the list of the highest $E_{\mathrm{A}}$ amplitudes leads to failure in the direct-methods application when customary $E$ estimates are employed for substructure solution. Overestimated Es stem from measurement errors in the MAD data. In this section, we try to inspect the overestimated reflections for systematic trends and investigate the reasons for the zero $\operatorname{SnB}$ success rates for the substructures that yielded at least one $S n B$ failure. Information such as the PDB code, data resolution (including both MAD data and $E_{\mathrm{A}}$ substructure data), number of reflections for $S n B$ phasing and reflection sorting is listed in the first four columns of Table 4 for the six selected substructures. Using $\left\langle E_{\mathrm{A}}\right\rangle$ as a reference, reflections that appear in the top $30 N_{\mu}$ (where $N_{\mu}$ is the number of Se sites) list of individual $E_{\mathrm{A}}$ estimates but do not appear in the top list of the averaged data set, $\left\langle E_{\mathrm{A}}\right\rangle$, are considered as overestimated reflections. For each set of two-wavelength estimates, the percentage of overestimated reflections and the correlation coefficient (CC) between individual estimates $E_{\mathrm{A}}$ and $\left\langle E_{\mathrm{A}}\right\rangle$ for all $30 N_{\mu}$ reflections and for overestimated reflections only are also listed in Table 4.

First of all, the percentage of overestimated $E$ s ranges from 25 to $50 \%$ and there is no correlation between the overestimated $E$ s and their averaged $\left\langle E_{\mathrm{A}}\right\rangle$ (indicated by near-zero $\mathrm{CC}$ values). For substructures $1 \mathrm{jxh}$ and 1eq2 outliers in the 3.6-3.1 and 3.4-3.0 $\AA$ resolution shells, respectively, are rejected by the $S H E L X C$ program, indicating poor quality of the integrated intensities in the resolution shell (perhaps owing to ice rings). The main reason for $S n B$ failures for these two substructures is the combination of low $E_{\mathrm{A}}$ data resolution and overestimated Es. For substructures 1cli, 118a, 1 hi 8 and $1 \mathrm{~m} 32$, a relatively low overall CC (bold numbers in column 6 of Table 4) from one of the three combinations indicates that one of the three-wavelength MAD data sets might have unacceptable quality (perhaps owing to radiation damage). For example, the overall CC from substructure 1hi8 indicates that the data quality of $E_{\mathrm{A}}\left(\lambda_{1}, \lambda_{3}\right)(\mathrm{CC}=0.236)$ is poorer than those of $E_{\mathrm{A}}\left(\lambda_{2}, \lambda_{3}\right)(\mathrm{CC}=0.360)$ or $E_{\mathrm{A}}\left(\lambda_{1}, \lambda_{2}\right)$ $(\mathrm{CC}=0.331)$. The $\operatorname{Sn} B$ success rates $[0.0,1.3$ and $41.3 \%$ for $E_{\mathrm{A}}\left(\lambda_{1}, \lambda_{3}\right), E_{\mathrm{A}}\left(\lambda_{2}, \lambda_{3}\right)$ and $E_{\mathrm{A}}\left(\lambda_{1}, \lambda_{2}\right)$, respectively] clearly indicate that the remote-wavelength anomalous difference had an unacceptable quality, possibly owing to radiation damage since four-wavelength MAD data were measured and the remote-wavelength data were measured last.

\section{Conclusions}

A new statistics-based procedure for substructure phasing using $F_{\mathrm{A}}$ formulae has been proposed and tested on $18 \mathrm{Se}-$ atom substructure examples. The procedure successfully identified overestimated reflections from $F_{\mathrm{A}}$ formulae to be a common cause of phasing failures and suggested effective ways to enhance the $F_{\mathrm{A}}$ estimation. The test results demonstrate that the improvements are significant, especially for 
those substructures previously deemed difficult to determine. Although the results were based on the Shake-and-Bake application, this new procedure can be applied to any directmethods-based substructure determination.

This research was supported by NIH grant GM072023. We thank everyone who made his or her SeMet MAD data available to us as test data.

\section{References}

Alphey, M. S., Bond, C. S., Tetaud, E., Fairlamb, A. H. \& Hunter, W. N. (2000). J. Mol. Biol. 300, 903-916.

Appleby, T. C., Erion, M. D. \& Ealick, S. E. (1999). Structure, 7, 629-641.

Appleby, T. C., Kinsland, C. L., Begley, T. P. \& Ealick, S. E. (2000). Proc. Natl Acad. Sci. USA, 97, 2005-2010.

Arjunan, P., Nemeria, N., Brunskill, A., Chandrasekhar, K., Sax, M., Yan, Y., Jordan, F., Guest, J. R. \& Furey, W. (2002). Biochemistry, 41, 5213-5221.

Butcher, S. J., Grimes, J. M., Makeyev, E. V. \& Bamford, D. H. (2001). Nature (London), 410, 235-240.

Chen, C. C. H., Zhang, H., Kim, A. D., Howard, A., Sheldrick, G. M., Dunaway-Mariano, D. \& Herzberg, O. (2002). Biochemistry, 41, 13162-13169.

Cheng, G., Bennett, E. M., Begley, T. P. \& Ealick, S. E. (2002). Structure, 10, 225-235.

Deacon, A. M., Ni, Y. S., Coleman, W. G. Jr \& Ealick, S. E. (2000). Structure, 8, 453-462.
Ekstrom, J. L., Mathews, I. I., Stanley, B. A., Pegg, A. E. \& Ealick, S. E. (1999). Structure, 7, 583-595.

Gulick, A. M., Lu, X. \& Dunaway-Mariano, D. (2004). Biochemistry, 43, 8670-8679.

Hendrickson, W. A., Smith, J. L. \& Sheriff, S. (1985). Methods Enzymol. 115, 41-55.

Istvan, E. S., Palnitkar, M., Buchanan, S. K. \& Deisenhofer, J. (2000). EMBO J. 19, 819-830.

Karle, J. (1980). Int. J. Quantum Chem. Symp. 7, 357-367.

Lamers, M. H., Perrakis, A., Enzlin, J. H., Winterwerp, H. H., De Wind, N. \& Sixma, T. K. (2000). Nature (London), 407, 711-717.

Li, C., Kappock, T. J., Stubbe, J., Weaver, T. M. \& Ealick, S. E. (1999). Structure, 7, 1155-1166.

Mathews, I. I., Erion, M. D. \& Ealick, S. E. (1998). Biochemistry, 37, 15607-15620.

Mathews, I. I., Kappock, T. J., Stubbe, J. \& Ealick, S. E. (1999). Structure, 7, 1395-1406.

McCarthy, A. A., Baker, H. M., Shewry, S. C., Patchett, M. L. \& Baker, E. N. (2001). Structure, 9, 637-646.

Miller, R., Gallo, S. M., Khalak, H. G. \& Weeks, C. M. (1994). J. Appl. Cryst. 27, 613-621.

Schneider, T. R. \& Sheldrick, G. M. (2002). Acta Cryst. D58, 17721779.

Sheldrick, G. M. (2008). Acta Cryst. A64, 112-122.

Turner, M. A., Yuan, C. S., Borchardt, R. T., Hershfield, M. S., Smith, G. D. \& Howell, P. L. (1998). Nature Struct. Biol. 5, 369-376.

Wang, W., Kappock, T. J., Stubbe, J. \& Ealick, S. E. (1998). Biochemistry, 37, 15647-15662.

Weeks, C. M. \& Miller, R. (1999). J. Appl. Cryst. 32, 120-124.

Xu, H. \& Hauptman, H. A. (2004). Acta Cryst. A60, 153-157.

Xu, H., Weeks, C. M. \& Hauptman, H. A. (2005). Acta Cryst. D61, 976-981. 